\title{
Waves of dissemination
}

DOI:

10.1038/nrmicro1470

\section{URLs}

Entrez Genome Project: http://www.ncbi.nlm.nih.gov/ entrez/query.

fcgi?db=genomeprj

Salmonella enterica

http://www.ncbi.nlm.nih. gov/entrez/query.fcgi?db=ge nomeprj\&cmd=Retrieve\&dop $\mathbf{t}=$ Overview\&list_uids $=232$

Yersinia pseudotuberculosis http://www.ncbi.nlm.nih. gov/entrez/query.fcgi?db=ge nomeprj\&cmd=Retrieve $\delta$ dop $\mathrm{t}=$ Overview\&list_uids $=16070$
Enteric pathogens are thought to cause systemic disease by ordered dissemination from the intestine to local lymph nodes and then to distant organs. There is, however, little evidence to support this model. Now, a report in The Journal of Experimental Medicine shows that colonization of the spleen and liver by Yersinia pseudotuberculosis does not require colonization of local lymph nodes, indicating that there might be an alternative pathway for the dissemination of enteric pathogens.

Similar to other enteric pathogens, such as Salmonella enterica serovar Typhimurium, ingestion of Y. pseudotuberculosis causes enteritis, and in mice, the bacteria also replicate in the liver and spleen, leading to death. So, Barnes and colleagues used oral inoculation of mice with Y. pseudotuberculosis as a model to study the pathways that are involved in systemic infection by enteric pathogens. On examining the kinetics of bacterial dissemination, they detected Y. pseudotuberculosis in the liver and spleen at both 30 minutes and 5 hours after inoculation, but the bacteria had been cleared by 11 hours. Y. pseudotuberculosis then reappeared in these tissues at 24 hours, and replication was well established at 72 hours, with the bacteria persisting until death. The authors postulated that these two temporally distinct waves of systemic dissemination might result from bacteria spreading by different routes.

To test their hypothesis, they studied strains of mice that lacked intestinal lymph nodes, thereby preventing dissemination through this route. They detected Y. pseudotuberculosis in the spleen and liver at late time points after inoculation ( 24 hours and later) but not at early time points (30 minutes and 5 hours), indicating that local lymph nodes have a crucial role in the early stage of systemic infection but not in the later stage, which results in lethal disease. These findings are supported by elegant experiments in which the clonal dissemination of oligonucleotide-tagged strains of Y. pseudotuberculosis was studied in wild-type mice. The authors showed that, at late time points after inoculation, bacterial clones in the liver and spleen were descendants of those present in the intestine but not of those in the intestinal lymph nodes, indicating that the bacteria that seed distant organs late in infection are derived directly from the intestinal pool.

In addition, the establishment of bacterial replication in distant organs (at 72 hours) was shown to occur after an increase in the bacterial load in the intestine, and oral administration of streptomycin (which is not well absorbed across the intestine) was shown to reduce the bacterial loads in both the intestine and distant organs. Together, these findings indicate that a replicating pool of bacteria in the intestine is a requirement for successful systemic infection.

There is, therefore, more than one route by which an enteric pathogen can cross the intestine and establish systemic disease. How this occurs in the absence of colonization of lymph nodes remains to be determined. Davina Dadley-Moore

\section{ORIGINAL RESEARCH PAPER Barnes, P. D.,} Bergman, M. A., Mecsas, J. \& Isberg, R. R. Yersinia pseudotuberculosis disseminates directly from a psering pseplicating poolin the intestine.J. Exp.Med. 203 1591-1601 (2006)

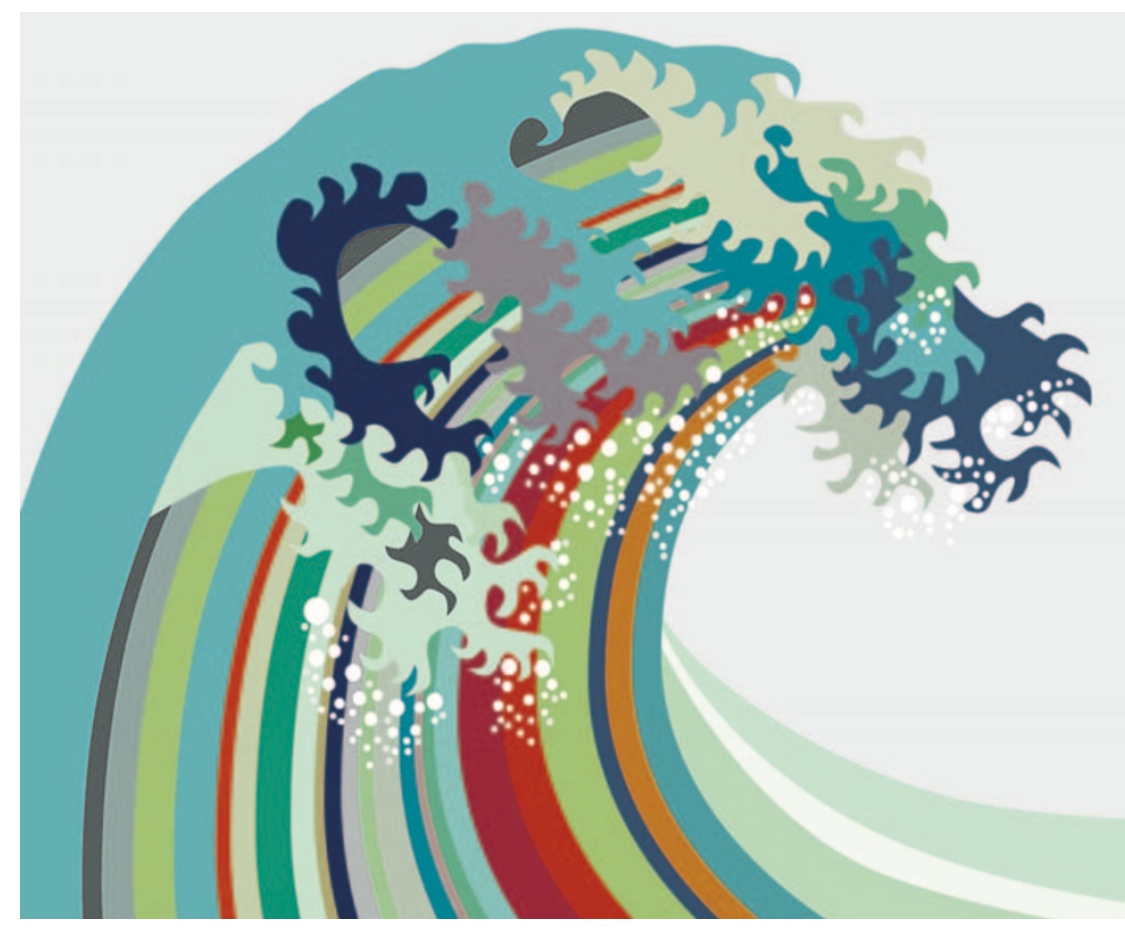

